\title{
Application of medicinal plants in several dermatovenerological entities
}

ŽELJAN MALES̆ ${ }^{1 *}$

DANIELA LEDIĆ DRVAR ${ }^{2}$

IVAN DUKA ${ }^{1}$

KRISTINA ŽUŽUL ${ }^{3}$

${ }^{1}$ University of Zagreb Faculty

of Pharmacy and Biochemistry

Department of Pharmaceutical Botany

10000 Zagreb, Croatia

${ }^{2}$ Department of Dermatology

and Venereology, University Hospital

Centre Zagreb, School of Medicine

University of Zagreb, 10000 Zagreb

Croatia

${ }^{3}$ Private Dermatology and Venereology

Clinic "Dr. Nada Videnić"

47000 Karlovac, Croatia

Accepted June 6, 2019

Published online July 8, 2019

\begin{abstract}
Treatment of skin conditions with medicinal plants has been an ongoing human activity lasting over thousands of years. The use of specific plant species developed regionally, based on local flora. Commonly used medicinal plants for dermatological complaints are: Phlebodium aureum (L.) J. Sm., Ginkgo biloba L., Rosmarinus officinalis L., Panax ginseng C.A.Mey., Allium cepa L., Aloe vera (L.) Burm.f., Capsicum annuum L., Berberis aquifolium Pursh, Camellia sinensis (L.) Kuntze, and Podophyllum peltatum $\mathrm{L}$.

The demand for complementary therapeutics is an emerging trend due to the awareness of potential side effects that synthetic drugs might cause. More scientific evidence and better documentation are needed before advising dermatologic patients on herbal medicinal treatment. Standardised extracts and formulations with proven clinical efficacy should be developed for this cause. Here provided review entails the use of herbal medicinal products in the treatment of frequent chronic skin diseases, such as vitiligo, alopecia, psoriasis and genital warts.
\end{abstract}

Keywords: medicinal plants, vitiligo, alopecia, psoriasis, genital warts

\section{INTRODUCTION}

Medicinal plants have been utilized for the treatment of various skin diseases in folk medicine. Species from local flora were used along with certain medicinal plants that were acquired through trade. In the recent years, research on medicinal plants and their bioactive compounds is growing in importance since chronic skin diseases, such as vitiligo, alopecia, psoriasis and genital warts, seem to be fairly resistant to the conventional treatment.

\section{Vitiligo}

Vitiligo is a common autoimmune pigmentation disorder affecting $0.5-4 \%$ of the population. Current treatment options include topical application of corticosteroids, calci-

\footnotetext{
*Correspondence; e-mail: zmales@pharma.hr
} 
neurin inhibitors, vitamin D analogues, phototherapy, laser therapy and surgery (1). Since the treatment results are often slow and unsatisfactory, with possible side effects, there is a great interest in complementary treatment modalities, such as medicinal plants.

Phlebodium aureum (L.) J. Sm. (Polypodium leucatomos Poir.), Polypodiaceae. - Dietary supplement containing $P$. aureum extract has been used for more than 30 years (1). Primary bioactive compounds are phenolic acids (2). When used in combination with narrow band (nb) UVB light (311 nm) or psoralen + UVA (PUVA) therapy, P. aureum supplementation may improve re-pigmentation and provide photoprotection (1). The results of a study which compared supplementation with $250 \mathrm{mg}$ of $P$. aureum three times daily in combination with nb-UVB to placebo and nb-UVB, re-pigmentation of head and neck area was achieved in $44 \%$ of patients in $P$. aureum group and in $27 \%$ of patients in the control group. P. aureum supplementation prevented oxidative damage, especially in lighter skin type patients with lower baseline antioxidant capacity (3). A similar study was conducted on 57 patients with generalized vitiligo, but the dosage of $P$. aureum was $480 \mathrm{mg}$ daily. Improvement was reported for $47.8 \%$ of the patients in the P. aureum and nb-UVB group, and $22 \%$ of patients with nb-UVB alone (4). The randomized study investigated the use of PUVA plus P. aureum, in comparison to placebo. More than $50 \%$ of re-pigmentation was reported for the patients in the P. aureum group. The threshold of $50 \%$ re-pigmentation was not achieved in the placebo group. Additionally, the immunomodulatory effect was shown by lymphocyte ratio normalization in the $P$. aureum group (5).

$P$. aureum demonstrated photoprotective activity in another study where the severity of erythema, pyrimidine dimer formation and epidermal proliferation were evaluated post-exposure to UV light. Pre-exposure supplementation with an oral dose of $7.5 \mathrm{mg} \mathrm{kg}^{-1}$ of $P$. aureum decreased the UV light-induced damage (6). The toxicity of oral administrated $P$. aureum was minimal, reported adverse reactions include mild pruritus and gastrointestinal upset (1).

Ginkgo biloba L., Ginkgoaceae. - G. biloba is a medicinal plant originating from Traditional Chinese Medicine shown to inhibit disease progression and augment re-pigmentation in vitiligo (1). The observed benefits are due to the anti-inflammatory, antioxidant and anxiolytic activity of flavonoids and terpene trilactones present in the leaves.

A double-blind study investigated the effects of supplementation with $40 \mathrm{mg} \mathrm{kg}^{-1}$ of G. biloba extract in patients with limited, slow-spreading vitiligo. A significant arrest of active disease was reported, re-pigmentation was achieved in $40 \%$ of treated patients compared with $9 \%$ of patients in the control group. A common side effect is gastrointestinal upset, but care should be taken because there have also been reports of haemorrhage and increased bleeding time (7).

\section{Alopecia}

Depending on its acuity and extent, hair loss is an important cause of anxiety.

Essential oils mixture. - A double-blind study included 86 patients with alopecia areata to investigate the efficacy of a mixture of essential oils as alopecia areata treatment. Selected essential oils for the mixture were obtained from: Thymus vulgaris L., Lavandula angustifolia Mill., Rosmarinus officinalis L. and Cedrus atlantica (Endl.) Manetti ex Carrière. 
The mixture was diluted in carrier oil, a combination of grape seed and jojoba oil. Prepared oily mixture was administered daily and massaged into the scalp. The control group followed the same routine using only carrier oil. The treatment group had statistically significant improvement by $44 \%$, based on expert and computerized analyses of sequential photographs, comparing to the control group with $15 \%$ improvement. There were no adverse effects reported (8).

Rosmarinus officinalis L., Lamiaceae. - R. officinalis is a well-known Mediterranean aromatic plant. The essential oil of $R$. officinalis improves microcirculation surrounding the hair follicle (9). Bioactive compounds of $R$. officinalis are phenolic acids, mainly caffeic and rosmarinic acid, and monoterpenoids like 1,8-cineole (10). A clinical study from 2015 compared the efficacy of rosemary essential oil to minoxidil $2 \%$ solution for the treatment of androgenetic alopecia. Patients used either minoxidil $2 \%$ solution $(n=50)$ or rosemary essential oil $(n=50)$. Significant increase in hair count was reported for both treatments without significant difference between the study groups. Scalp irritation was more frequent in minoxidil $2 \%$ solution group (9).

Panax ginseng C. A. Mey., Araliaceae. - Ginsenosides are saponin compounds derived from $P$. ginseng with a wide spectrum of biological activity, with the influence on hairgrowth being only one of the proposed activities (11). In 2014, a study was conducted in order to assess the efficacy of $P$. ginseng supplementation in treating female pattern alopecia. Group 1 consisted of patients who used topical $3 \%$ minoxidil solution, while group 2 used topical $3 \%$ minoxidil solution and oral $P$. ginseng supplement. An expert panel of dermatologists concluded that group 2 showed significantly better results although the hair density and thickness were improved in both groups (12).

Allium cepa L., Amaryllidaceae. - Sulphurous and phenolic compounds from onion juice act as irritants, thereby causing mild dermatitis which might provoke hair growth. A study where $A$. cepa juice was used as a treatment for alopecia areata has shown significantly more hair re-growth after six months of the treatment (86.9\%). The control group used only tap water and $13 \%$ of hair re-growth was reported (13).

\section{Psoriasis}

Most studied medicinal preparations for the treatment of psoriasis are Aloe vera juice, capsaicin, indigo naturalis and Berberis aquifolium extracts (14).

Aloe vera (L.) Burm.f., Liliaceae. - A. vera has been used for medicinal purposes for more than 2000 years. Gel-like leaf juice of $A$. vera is a pharmaceutical material with reported antiinflammatory, antipruritic, and wound healing effects. Bioactive compounds of the juice are phenolics, polysaccharides, organic acids and various other metabolites (15).

A study with a group of patients who were treated with $0.5 \%$ of $A$. vera juice in hydrophilic vehicle showed statistically significant improvement in the $A$. vera group compared to the group treated with hydrophilic vehicle exclusively. The $A$. vera juice caused a decrease in the severity of symptoms in $83.3 \%$ of patients (16).

Capsicum annuum L., Solanaceae. - The main active ingredient of pepper extracts for topical application is capsaicin which inhibits transcription factors NF- $\kappa$ B and AP-1 
activation in vitro (17). Substance P could play a role in neurogenic pathophysiology of psoriasis. In psoriatic plaques, substance P-activated inflammatory pathways can cause vasodilation and angiogenesis, followed by keratinocyte hyperproliferation. Capsaicin causes an efflux of substance $P$ from sensory neurons by activating vanilloid receptors and thereby depleteing cutaneous substance P (14).

Topically used $0.025 \%$ capsaicin cream has shown to improve symptoms like scaling and erythema in patients with moderate and severe psoriasis after 6 weeks of treatment (18). In another study, with the same dosage and duration of the treatment, along with improvements in scaling and erythema a significant decrease in thickness and pruritus are reported. The application of capsaicin is followed by an intense and short-lasting burning sensation (19).

Indigo naturalis. - Indigo naturalis is a preparation from Traditional Chinese Medicine usually obtained from plant species Strobilanthes cusia (Nees) Kuntze, Acanthaceae, and has long been used as a therapy for psoriasis and other conditions with an inflammatory component. Indirubin was identified as the active compound, besides the anti-inflammatory effect, inhibition of proliferation and promotion of differentiation in epidermal keratinocytes are proposed mechanisms of action (14).

Indigo naturalis ointment was studied as a topical therapy in moderate plaque psoriasis. After 8 weeks of randomized, double-blind, placebo-controlled clinical study, the results were quantified through Psoriasis Area Severity Index (PASI). Significant improvement by $75 \%$ (PASI 75) had been achieved in $56.3 \%$ of patients compared to $0.0 \%$ in the placebo group. Additionally, in order to establish the exact mechanism, a biopsy was preformed showing the down-regulation of the IL-17 pathway (20). Lin et al. (21) reported a reduction of plaque area and significant improvement in symptoms after the treatment with an ointment containing indigo naturalis. In another study by Lin et al. (22) with the same indigo preparation, the PASI 75 was achieved in $74 \%$ of patients in the treated group. Temporary erythema and itching on the application site were noted as adverse effects. Oily extract of indigo naturalis was formulated for a clinical study assessing the topical application in nail psoriasis and a significant improvement in the severity of symptoms was quantified with Nail Psoriasis Severity Index (NAPSI) after 12 and 24 weeks of treatments, without adverse effects (23).

Berberis aquifolium Pursh (Mahonia aquifolium (Pursh) Nutt.), Berberidaceae. - B. aquifolium use for the treatment of psoriasis is linked to the presence of berberine, an antiinflammatory alkaloid that reduces keratinocyte hyperproliferation markers typical for psoriasis $(14,24)$.

The extract of B. aquifolium formulated into a cream was tested in a clinical study for the treatment of chronic plaque psoriasis. Improvements in PASI score were significant in comparison to placebo cream (24). In another study, B. aquifolium extract was compared to conventional therapeutics calcipotriol and tazarotene; $B$. aquifolium extract was either as effective or better in treating disease symptoms (14).

\section{Condyloma accuminata}

Camellia sinensis (L.) Kuntze, Theaceae. - C. sinensis is a rich source of polyphenols. Epigallocatechin and epigallocatechin gallate are the most abundant polyphenols but the 
composition might vary depending on leaf processing method (25). Green tea polyphenols have been shown to possess antioxidant, antiproliferative, proapoptotic, antiviral and antitumor properties (26).

Ointment with green tea extract, comprised of more than $85 \%$ catechins, is a patientadministered therapy for external genital warts. Phase III clinical trials demonstrated significantly higher efficacy and lower recurrence rates compared to other currently available patient-applied treatments. In two identically designed, randomized, doubleblind, Phase III trials, a total of 1005 participants (535 men and 470 women) were included to receive $15 \%$ green tea extract ointment, $10 \%$ green tea extract ointment or vehicle. Treatment was applied at 8-hour intervals three times a day for 16 weeks or until complete resolution of all baseline warts was observed. Rates of complete clearance of all warts in both green tea extract ointment groups were significantly superior to vehicle $\left(P_{S}<0.001\right)$. The median time to complete wart clearance in the two trials was 16 weeks and 10 weeks in the $15 \%$ and $10 \%$ green tea extract ointment groups, respectively. In all studies, recurrence rates were low and complete clearance rates were higher in women than in men (27).

Podophyllum peltatum L., Berberidaceae. - P. peltatum has been used for centuries in the treatment of warts and skin cancers by indigenous people of the Americas. Podophyllin is a resin extracted from the root of the P. peltatum (28). When treating genital warts, a recent study showed that a combination of cryotherapy and podophyllin therapy is more efficient than cryotherapy alone, shortening the treatment regimen (29). According to a meta-analysis of 9 randomised controlled trials, podophyllotoxin, the main ingredient of podophyllin, in the concentration of $0.5 \%$ caused complete clearance of genital warts in $56.41 \%$ of patients. Burning sensation, pain, pruritus and inflammation might occur on the site of application (28).

\section{CONCLUSIONS}

Medicinal plants have been used for the treatment of skin conditions long before synthetic drugs came on the market. Advantages of synthetic drugs for prescribers are reliable scientific background, quality and stability of the product, which is ensured by legislative means. Although herbal medicinal products can only strive to achieve a quality level comparable to conventional therapy, certain advantages cannot be ignored. Firstly, described medicinal plants have a better safety profile, with the exception of Ginkgo conventional therapeutic for indications other than vitiligo, with well-known side-effects and potential interactions. Secondly, multiple pharmacological targets are modulated with a complex mixture of plant metabolites, thereby providing a possible solution for diseases resistant to conventional therapy.

\section{REFERENCES}

1. B. E. Cohen, N. Elbuluk, E. W. Mu and S. J. Orlow, Alternative Systemic Treatments for Vitiligo: A Review, Am. J. Clin. Dermatol. 16 (2015) 463-474; https://doi.org/10.1007/s40257-015-0153-5

2. F. Garcia, J. P. Pivel, A. Guerrero, A. Brieva, M. P. Martinez-Alcazar, M. Caamano-Somoza and S. Gonzalez, Phenolic components and antioxidant activity of Fernblock, an aqueous extract of the aerial parts of the fern Polypodium leucotomos, Methods Find Exp. Clin. Pharmacol. 28 (2006) 157-160; https://doi.org/10.1358/mf.2006.28.3.985227 
3. S. M. A. Middelkamp-Hup, J. D. Bos, F. Rius-Diaz, S. Gonzalez and W. Westerhof, Treatment of vitiligo vulgaris with narrow-band UVB and oral Polypodium leucotomos extract: a randomized double-blind placebo-controlled study, J. Eur. Acad. Dermatol. Venereol. 21 (2007) 942-950; https:// doi.org/10.1111/j.1468-3083.2006.02132.x

4. A. Pacifico, A. Vidolin, G. Leone and P. Iacovelli, Combined treatment of narrowband ultraviolet B light (NBUVB) phototherapy and oral Polypodium leucotomos extract versus NB UVB phototherapy alone in the treatment of patients with vitiligo, J. Am. Acad. Dermatol. 60 (2009) AB154; https://doi. org/10.1016/j.jaad.2008.11.676

5. E. Reyes, P. Jaen, E. de las Heras, F. Carrion, M. Alvarez-Mon, E. de Eusebio, M. Alvare, J. Cuevas, S. González and V. G. Villarrubia, Systemic immunomodulatory effects of Polypodium leucotomos as an adjuvant to PUVA therapy in generalized vitiligo: a pilot study, J. Dermatol. Sci. 41 (2006) 213-216; https://doi.org/10.1016/j.jdermsci.2005.12.006

6. M. A. Middelkamp-Hup, M. A. Pathak, C. Parrado, D. Goukassian, F. Rius-Diaz, M. C. Mihm, T. B. Fitzpatrick, and S. González, Oral Polypodium leucotomos extract decreases ultraviolet-induced damage of human skin, J. Am. Acad. Dermatol. 51 (2004) 910-918; https://doi.org/10.1016/j. jaad.2004.06.027

7. P. D. Shenefelt, Herbal Treatment for Dermatologic Disorders, in Herbal Medicine: Biomolecular and Clinical Aspects, $2^{\text {nd }}$ ed., CRC Press/Taylor \& Francis, Boca Raton (FL) 2011, Chapter 18.

8. I. C. Hey, M. Jamieson and A. D. Ormerod, Randomized trial of aromatherapy, Arch. Dermatol. 134 (1998) 1349-1352.

9. Y. Panahi, M. Taghizadeh, E. T. Marzony and A. Sahebkar, Rosemary oil vs minoxidil $2 \%$ for the treatment of androgenetic alopecia: a randomized comparative trial, Skinmed. 13 (2015) 15-21.

10. M. Y. Dhariwala and P. Ravikumar, An overview of herbal alternatives in androgenetic alopecia, J. Cosmet. Dermatol. (2019); https://doi.org/10.1111/jocd.12930

11. B. Y. Choi, Hair-growth potential of ginseng and its major metabolites: A review on its molecular mechanisms, Int. J. Mol. Sci. 19 (2018) E2703; https://doi.org/ 10.3390/ijms19092703

12. H. J. Ryu, M. G. Yoo and S. W. Son, The efficacy of $3 \%$ minoxidil vs. combined $3 \%$ minoxidil and Korean red ginseng in treating female pattern alopecia, Int. J. Dermatol. 53 (2014) 340-342; https:// doi.org/10.1111/ijd.12359

13. K. E. Sharquie and H. K. Al-Obaidi, Onion juice (Allium cepa L.), a new topical treatment for alopecia areata, J. Dermatol. 29 (2002) 343-346; https://doi.org/10.1111/j.1346-8138.2002.tb00277.x

14. B. Farahnik, D. Sharma, J. Alban and R. K. Sivamani, Topical Botanical Agents for the Treatment of Psoriasis: A Systematic Review, Am. J. Clin. Dermatol. 18 (2017) 451-468; https://doi.org/10.1007/ s40257-017-0266-0

15. M. H. Radha and N. P. Laxmipriya, Evaluation of biological properties and clinical effectiveness of Aloe vera: A systematic review, J. Tradit. Complement. Med. 5 (2015) 21-26; https://doi.org/10.1016/j. jtcme.2014.10.006

16. T. A. Syed, S. A. Ahmad, A. H. Holt, S. H. Ahmad and M. Azfal, Management of psoriasis with Aloe vera extract in a hydrophilic cream: a placebo-controlled, double-blind study, Trop. Med. Int. Health 1 (1996) 505-509.

17. Y. J. Surh, S. H. Seoung, Y. S. Keum, H. J. Seo and S. L. Sang, Inhibitory effects of curcumin and capsaicin on phorbol ester-induced activation of eukaryotic transcription factors, NF- $\kappa$ B and AP1, Biofactors. 12 (2000) 107-112.

18. J. E. Bernstein, L. C. Parish, M. Rapaport, M. M, Rosenbaum and H. H. Jr. Roenigk, Effects of topically applied capsaicin on moderate and severe psoriasis vulgaris, J. Am. Acad. Dermatol. 14 (1986) 504-507.

19. C. N. Ellis, B. Berberian, V. I. Sulica, W. A. Dodd, M. T. Jarratt, H. I. Katz, S. Prawer, G. Krueger, I. H. Jr. Rex and J. E. Wolf, A double-blind evaluation of topical capsaicin in pruritic psoriasis, J. Am. Acad. Dermatol. 29 (1993) 438-442. 
20. H. M. Cheng, Y. C. Wu, Q. Wang, M. Song, J. Wu, D. Chen, K. Li, E. Wadman, S-T. Kao, T-C. Li, F. Leon, K. Hayden, C. Brodmerkel and C. C. Huang, Clinical efficacy and IL-17 targeting mechanism of Indigo naturalis as a topical agent in moderate psoriasis, BMC Complement Altern Med. 17 (2017) 439; https://doi.org/10.1186/s12906-017-1947-1

21. Y. K. Lin, W. R. Wong, Y. C. Chang, C. J. Chang, P. K. Tsay, S. C. Chang and J. H. Pang, The efficacy and safety of topically applied indigo naturalis ointment in patients with plaque-type psoriasis, Dermatology. 214 (2007) 155-161; https://doi.org/10.1159/000098576

22. Y. K. Lin, C. J. Chang, Y. C. Chang, W. R. Wong, S. C. Chang and J. H. Pang, Clinical assessment of patients with recalcitrant psoriasis in a randomized, observerblind, vehicle-controlled trial using indigo naturalis, Arch. Dermatol. 144 (2008) 1457-1464; https://doi.org/10.1001/ archderm.144.11.1457

23. Y. K. Lin, L. C. See, Y. H. Huang, Y. C. Chang, T. C. Tsou, T. Y. Lin and N. L. Lin, Efficacy and safety of Indigo naturalis extract in oil (Lindioil) in treating nail psoriasis: a randomized, observerblind, vehicle-controlled trial, Phytomedicine 21 (2014) 1015-1020; https://doi.org/10.1016/j. phymed.2014.02.013

24. S. Bernstein, H. Donsky, W. Gulliver, D. Hamilton, S. Nobel and R. Norman, Treatment of mild to moderate psoriasis with Relieva, a Mahonia aquifolium extract: a double-blind, placebo-controlled study, Am. J. Ther. 13 (2006) 121-126.

25. S. M. Meltzer, B. J. Monk and K. S. Tewari, Green tea catechins for treatment of external genital warts, Am. J. Obstet. Gynecol. 200 (2009); https://doi.org/10.1016/j.ajog.2008.07.064

26. C. S. Yang and H. Wang, Cancer Preventive Activities of Tea Catechins, Molecules 21 (2016); https:// doi.org/10.3390/molecules21121679

27. A. K. Gupta and D. Daigle, Sinecatechins 10\% ointment: a green tea extract for the treatment of external genital warts, Skin Therapy Lett. 20 (2015) 6-8.

28. S. Colantonio and J. K. Rivers, Botanicals with dsermatologic properties derived from first nations gealing: Part 2-plants and algae, J. Cutan. Med. Surg. 21 (2017) 299-307; https://doi. org/10.1177/1203475416683390

29. N. Sharma, S. Sharma and C. Singhal, A comparative study of liquid nitrogen cryotherapy as monotherapy versus in combination with podophyllin in the treatment of condyloma acuminata, J. Clin. Diagn. Res. 11 (2017) WC01-WC05; https://doi.org/10.7860/JCDR/2017/23797.9339 